\title{
Solvent and substituent effect on electronic spectra of $N$-(4-substituted phenyl)-2,3-diphenylpropanamides
}

\author{
Nataša Valentić, Dušan Mijin, Gordana Ušćumlić," Aleksandar Marinković, \\ and Slobodan Petrović \\ Department of Organic Chemistry, Faculty of Technology and Metallurgy, University of \\ Belgrade, Karnegijeva 4, P.O. Box 3503, 11120 Belgrade, Serbia \\ E-mail: goca@tmf.bg.ac.yu
}

\begin{abstract}
The UV absorption spectra (200-400 nm) of eleven $N$-(4-substituted phenyl)-2,3-diphenylpropanamides 1 have been recorded in fourteen solvents of different polarity. A simple Hammett equation was used to study the effects of substituents on the UV spectra of compounds $\mathbf{1}$. The effects of solvent polarity and hydrogen bonding on the absorption spectra are interpreted by means of linear solvation energy relationships. The results show that the solvent effect on UV absorption spectra of the investigated amides are very complex and strongly depends on the nature of the substituents of the phenyl ring.
\end{abstract}

Keywords: Absorption spectra, diphenylpropanamide, simple Hammett equation, solvent polarity, substituent effect, linear solvation energy relationship

\section{Introduction}

In our previous work we have reported the correlations between ultraviolet absorption frequencies of 3-N-(4-substituted phenyl)-5-carboxyuracils, ${ }^{1,2} 5$-substituted 3-cyano-6-hydroxy4-methyl-2-pyridones, ${ }^{3}$ 3-( $N$-alkyl)-5-carboxyuracils, ${ }^{4}$ 4,6-disubstituted 3-cyano-2-pyridones, ${ }^{5}$ and also the correlations between UV-Vis absorption frequencies and substituent and solvent parameters of 5-(4-substituted arylazo)-3-cyano-6-hydroxy-4-methyl-2-pyridones ${ }^{6}$ and 5-(4substituted arylazo)-3-cyano-4,6-dimethyl-2-pyridones. ${ }^{7}$ In addition, the transmission of electronic effects of substituents through the amide bond of $N$-(4-substituted phenyl)-2phenylacetamides $^{8}$ has been reported, and the effect of substituents on the IR spectral characteristics of the $\mathrm{C}=\mathrm{O}$ group has been investigated. Later, $N$-(4-substituted phenyl)benzamides ${ }^{9}$ and $N$-(4-substituted phenyl)-2-phenylacetamides have been compared with respect to the correlation between UV absorption frequencies and solvent parameters. 
In this work we report the synthesis of five new $N$-(4-substituted phenyl)-2,3-diphenylpropanamides 1a,g-j (Figure 1), the UV absorption spectra (200-400 nm) of eleven compounds $\mathbf{1 a}-\mathbf{k}$ recorded in fourteen solvents of different polarity, and the study of the effects of substituents and solvents on the UV spectra.

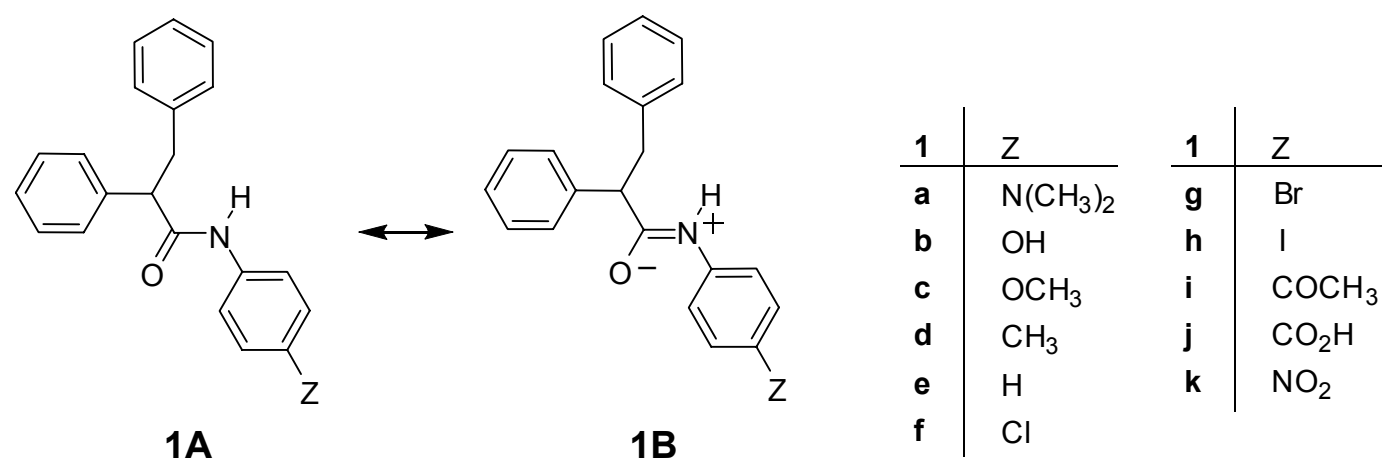

Figure 1. Canonical structures 1A and 1B of $N$-(4-substituted phenyl)-2,3-diphenylpropanamides $\mathbf{1 a}-\mathbf{k}$.

\section{Results and Discussion}

The main goal of this work is to explain the effects of the substituents in the UV spectrum of $N$-(4-substituted phenyl)-2,3-diphenylpropanamides 1 in different solvents with reference to $N$-phenyl-2,3-diphenylpropanamide 1 e $(\mathrm{Z}=\mathrm{H})$. In the solvents used two absorption bands were found in the UV spectra of compounds 1a-k; the lower energy band is sensitive to the electronic properties of the substituent (Table 1). No correlation was found for the high energy band.

The data in Table 1 confirm that the position of the UV absorption frequency depends on the nature of the N-phenyl substituent. A 4-substituent in the benzene ring causes a bathochromic shift of the long-wavelength absorption maximum as compared to the unsubstituted amide.

In order to explain the trend shown in Table 1, the wavenumbers were correlated with various sets of substituent parameters $\sigma$. The plot of the absorption frequencies $v s \sigma_{\mathrm{p}}$ or $\sigma_{\mathrm{p}}{ }^{+}$and $\sigma_{\mathrm{p}}{ }^{-}$constants gives correlations which show deviations from the Hammett equation in all investigated solvents (Figure 2). 
Table 1. UV spectral data of $N$-(4-substituted phenyl)-2,3-diphenylpropanamides 1a-k

\begin{tabular}{|c|c|c|c|c|c|c|c|c|c|c|c|}
\hline \multirow{2}{*}{$\mathrm{Z}:$} & \multicolumn{11}{|c|}{$\widetilde{v}_{\max } \times 10^{-3}\left[\mathrm{~cm}^{-1}\right]$} \\
\hline & $\mathrm{N}\left(\mathrm{CH}_{3}\right)_{2}$ & $\mathrm{OH}$ & $\mathrm{OCH}_{3}$ & $\mathrm{CH}_{3}$ & $\mathrm{H}$ & $\mathrm{Cl}$ & $\mathrm{Br}$ & I & $\mathrm{COCH}_{3}$ & $\mathrm{CO}_{2} \mathrm{H}$ & $\mathrm{NO}_{2}$ \\
\hline Methanol & 36.42 & 41.02 & 39.68 & 40.82 & 41.15 & 40.06 & 40.82 & 39.56 & 35.01 & 38.20 & 31.77 \\
\hline Ethanol & 36.21 & 40.00 & 40.13 & 40.58 & 41.15 & 40.00 & 40.36 & 39.46 & 34.82 & 37.88 & 31.61 \\
\hline 1-Propanol & 36.18 & 40.10 & 39.59 & 40.10 & 40.32 & 40.00 & 39.90 & 38.91 & 34.92 & 36.79 & 31.67 \\
\hline 2-Propanol & 36.82 & 40.45 & 39.49 & 40.19 & 41.36 & 39.97 & 40.13 & 39.78 & 34.51 & 37.91 & 31.61 \\
\hline 1-Butanol & 36.26 & 40.29 & 40.00 & 40.13 & 41.22 & 40.26 & 40.13 & 39.43 & 35.54 & 38.02 & 31.69 \\
\hline $\begin{array}{l}\text { 2-Methyl-2- } \\
\text { propanol }\end{array}$ & 35.21 & 38.46 & 38.85 & 41.02 & 42.05 & 38.26 & 37.85 & 38.05 & 33.67 & 36.90 & 31.65 \\
\hline Chloroform & 36.71 & 39.65 & 39.15 & 39.65 & 39.31 & 39.37 & 38.70 & 38.94 & 37.97 & 38.05 & 32.03 \\
\hline Dioxan & 36.00 & 38.70 & 38.31 & 38.73 & 38.73 & - & 37.74 & 37.82 & 35.24 & 36.36 & 32.79 \\
\hline Diethyl eter & 36.60 & 40.23 & 39.78 & 40.32 & 40.42 & 40.39 & 39.94 & 39.68 & 36.52 & 37.51 & 32.92 \\
\hline Ethyl acetate & 35.92 & 38.91 & 38.76 & 38.91 & 38.79 & 38.79 & 38.49 & 38.49 & 35.29 & 36.82 & 31.81 \\
\hline Acetonitrile & 37.51 & 40.32 & 39.65 & 40.45 & 41.32 & 40.19 & 40.45 & 39.97 & 37.34 & 38.73 & 31.57 \\
\hline DMF & 35.61 & 37.12 & 37.09 & 37.17 & 36.98 & 37.17 & 36.90 & 36.98 & 33.92 & 35.71 & 30.73 \\
\hline DMA & 35.46 & 37.31 & 37.06 & 37.09 & 37.29 & 37.12 & 36.82 & 36.93 & 34.11 & 35.59 & 30.67 \\
\hline DMSO & 35.36 & 37.91 & 37.48 & 37.76 & 37.26 & 37.62 & 36.95 & 36.90 & 33.85 & 35.29 & 30.40 \\
\hline
\end{tabular}

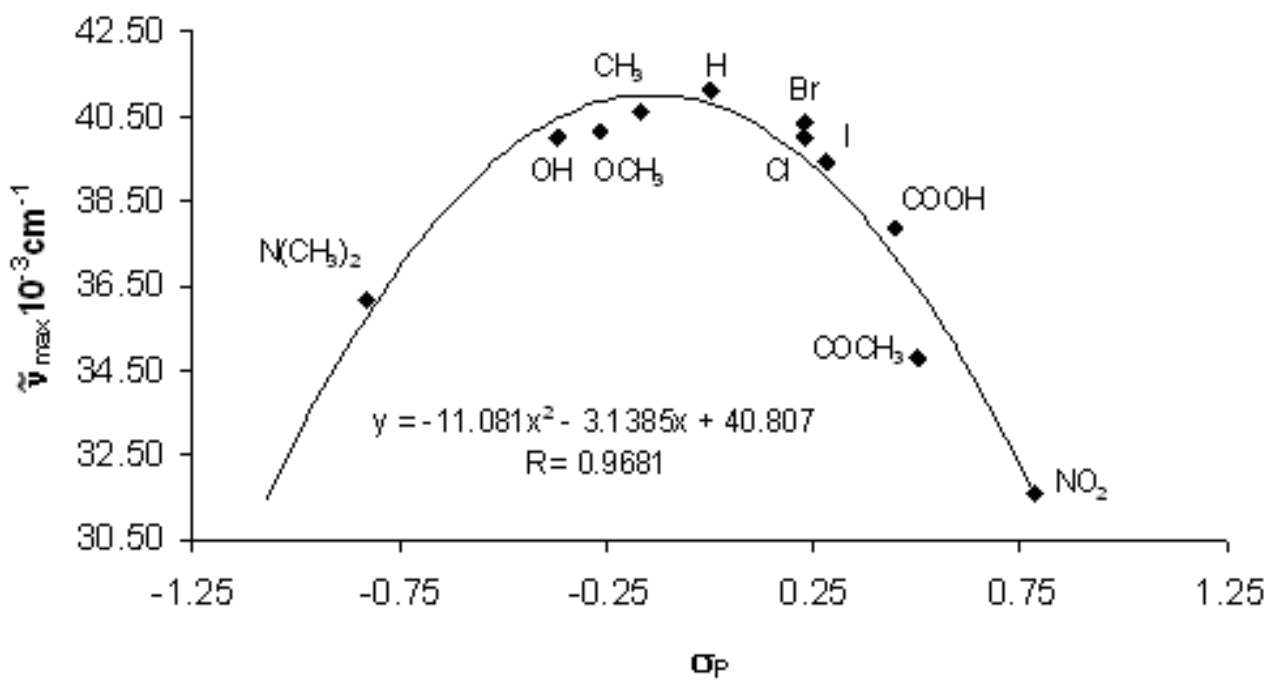

Figure 2. Relationship between $\widetilde{v}_{\max }$ and $\sigma_{\mathrm{p}}$ substituent constants for $N$-(4-substituted phenyl)2,3-diphenylpropanamides $\mathbf{1}$ in ethanol.

With electron-releasing substituents attached to the N-phenyl group of $\mathrm{N}$-(4-substituted phenyl)-2,3-diphenylpropanamides (structure 1C) the substituent effects are transmitted through the amide bond by the usual mechanism. 


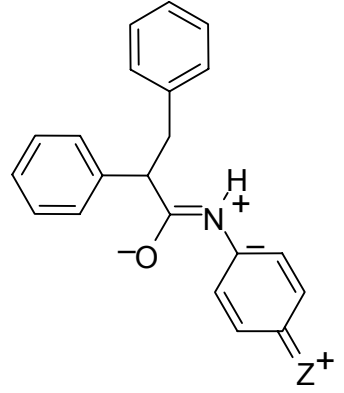

$1 C$

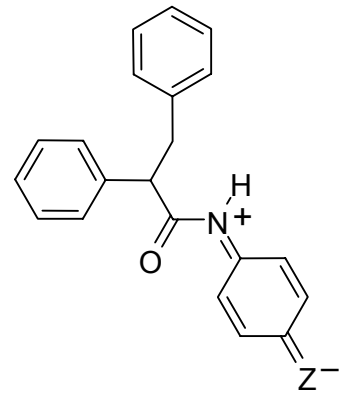

1D

Figure 3. Resonance effect of electron-releasing (structure 1C) and electron-withdrawing (structure 1D) substituents in 4-position of $\mathrm{N}$-phenyl-substituted-2,3-diphenylpropanamides $\mathbf{1}$.

However, the effect of electron-withdrawing substituents (structure 1D) appears to be quite opposite. The reversal of the substituent effect in the investigated system is interpreted by assuming a competition between the local $\pi$-polarization within a strongly conjugated amide group and the electron-donating mesomerism in the anilino group.

Electron-donating substituents increase the electron density at the anilide nitrogen atom, decrease the $\mathrm{n}-\pi^{*}$ transition energy and produce bathochromic shifts of the long wavelength absorption maximum as compared to that of $\mathrm{N}$-phenyl-2,3-diphenylpropanamide.

By contrast, electron-withdrawing substituents can be expected to decrease the electron density at the anilide nitrogen atom. However, our results show that these substituents also produce bathochromic shifts. The effect of electron-withdrawing substituents is opposite to the effect of the local polarization in the amide group and causes a similar effect on the UV absorption maxima as electron-donating substituents. These results are in accordance with results previously obtained for hydantoin derivatives, ${ }^{10} \mathrm{~N}$-(4-substituted phenyl) benzamides, ${ }^{9}$ and $N$-(4substituted phenyl)-2- phenylacetamides. ${ }^{8}$

The effect of solvent polarity and hydrogen bonding on $N$-(4-substituted phenyl)-2,3diphenylpropanamides 1 are interpreted by the linear solvation energy relationship ${ }^{11}$ using a Kamlet-Taft general solvatochromic equation of the following form:

$$
v=v_{\mathrm{o}}+\mathrm{s} \pi^{*}+\mathrm{b} \beta+\mathrm{a} \alpha
$$

where $\pi^{*}$ is a measure of the solvent dipolarity/polarizability, $\beta$ is the scale of the solvent hydrogen bond acceptor basicity, $\alpha$ is the scale of the solvent hydrogen bond donor acidity, and $v_{0}$ is the regression value of the solute property in the reference solvent cyclohexane. The regression coefficients $\mathrm{s}, \mathrm{b}$ and $\mathrm{a}$ in Eq.1 measure the relative susceptibilities of the solvent dependant solute property (absorption frequencies) to the indicated solvent parameters. 
Table 2. Solvent parameters ${ }^{12}$

\begin{tabular}{clccc}
\hline No. & Solvent & $\pi^{*}$ & $\beta$ & $\alpha$ \\
\hline 1 & Methanol & 0.6 & 0.62 & 0.93 \\
2 & Ethanol & 0.6 & 0.62 & 0.93 \\
3 & 1-Propanol & 0.54 & 0.77 & 0.83 \\
4 & 2-Propanol & 0.52 & 0.83 & 0.78 \\
5 & 1-Butanol & 0.48 & 0.95 & 0.76 \\
& 2-Methyl-2- & 0.47 & 0.88 & 0.79 \\
6 & propanol & & & \\
7 & Chloroform & 0.41 & 1.01 & 0.68 \\
\hline
\end{tabular}

\begin{tabular}{clccc}
\hline No. & Solvent & $\pi^{*}$ & $\beta$ & $\alpha$ \\
\hline 8 & Dioxan & 0.58 & 0 & 0.44 \\
9 & Diethyl eter & 0.55 & 0.37 & 0 \\
10 & Ethyl acetate & 0.27 & 0.47 & 0 \\
11 & Acetonitrile & 0.55 & 0.45 & 0 \\
12 & DMF & 0.75 & 0.31 & 0.19 \\
13 & DMA & 0.88 & 0.69 & 0 \\
14 & DMSO & 0.88 & 0.76 & 0 \\
\hline
\end{tabular}

The solvent parameters ${ }^{12}$ are shown in Table 2. The correlations of the spectroscopic data were carried out by means of multiple linear regression analysis. It was found that the absorption frequencies for investigated amides in twelve selected solvents (Table 3) show satisfactory correlation with $\pi^{*}, \alpha$ and $\beta$ parameters. The results of the multiple regressions are presented in Tables 3 and 4 . The negative sign of $s$ and $b$ coefficients for all amides (Table 3 ) indicate a bathochromic shifts with both increasing solvent dipolarity/polarizability and solvent hydrogenbond acceptor basicity. This suggests stabilization of the electronically excited state relative to the ground state. The positive sign of a coefficient for all amides indicates a hypsochromic shifts with increasing hydrogen-bond donor acidity of the solvent. This suggests stabilization of the ground state relative to the electronically excited state. The percentage contributions of the solvatochromic parameters (Table 4) for amides with a strong electron-donating substituent as the dimethylamino group and a strong electron-attracting substituent as the nitro group show that a major contribution of the solvatochromism is due to solvent dipolarity/polarizability. These results are in accordance with canonical structures 1C and 1D, and their stabilization is accounted to the solvent dipolarity/polarizability (non-specific solute-solvent interactions) rather than to solvent basicity and acidity properties. The percentage contributions of solvatochromic parameters for amides with moderate electron-donating and electron-attracting substituents in the phenyl group show that most of the solvatochromism is due to solvent basicity and acidity properties (specific solute-solvent interactions). 
Table 3. Regression fits to solvatochromic parameters

\begin{tabular}{|c|c|c|c|c|c|c|c|c|}
\hline $\begin{array}{c}\text { Substituent } \\
\mathrm{X} \\
\end{array}$ & $\begin{array}{c}v_{o} \\
\left(10^{3} \mathrm{~cm}^{-1}\right)\end{array}$ & $\begin{array}{c}S \\
\left(10^{3} \mathrm{~cm}^{-1}\right)\end{array}$ & $\begin{array}{c}b \\
\left(10^{3} \mathrm{~cm}^{-1}\right)\end{array}$ & $\begin{array}{c}a \\
\left(10^{3} \mathrm{~cm}^{-1}\right)\end{array}$ & $\mathrm{R}^{\mathrm{a}}$ & $\mathrm{s}^{\mathrm{b}}$ & $\mathrm{F}^{\mathrm{c}}$ & $\begin{array}{c}\text { Solvents used in } \\
\text { correlation }^{\mathrm{d}}\end{array}$ \\
\hline $\mathrm{N}\left(\mathrm{CH}_{3}\right)_{2}$ & $\begin{array}{c}37.11 \\
( \pm 0.27)\end{array}$ & $\begin{array}{c}-1.39 \\
( \pm 0.39)\end{array}$ & $\begin{array}{c}-0.52 \\
( \pm 0.30)\end{array}$ & $\begin{array}{c}0.53 \\
( \pm 0.21)\end{array}$ & $\begin{array}{c}0.9 \\
11\end{array}$ & 0.23 & 13 & $1,2,3,4,5,7,8,9,10,12,13,14$ \\
\hline $\mathrm{OH}$ & $\begin{array}{c}42.28 \\
( \pm 0.88)\end{array}$ & $\begin{array}{c}-1.92 \\
( \pm 0.98)\end{array}$ & $\begin{array}{c}-4.12 \\
( \pm 1.05)\end{array}$ & $\begin{array}{c}2.80 \\
( \pm 0.62)\end{array}$ & $\begin{array}{l}0.9 \\
22\end{array}$ & 0.61 & 15 & $1,2,3,4,5,6,9,1011,12,13,14$ \\
\hline $\mathrm{OCH}_{3}$ & $\begin{array}{c}41.67 \\
( \pm 0.61)\end{array}$ & $\begin{array}{c}-2.52 \\
( \pm 0.68)\end{array}$ & $\begin{array}{c}-2.81 \\
( \pm 0.73)\end{array}$ & $\begin{array}{c}2.02 \\
( \pm 0.43)\end{array}$ & $\begin{array}{l}0.9 \\
48\end{array}$ & 0.42 & 24 & $1,2,3,4,5,6,9,1011,12,13,14$ \\
\hline $\mathrm{CH}_{3}$ & $\begin{array}{c}42.37 \\
( \pm 0.90)\end{array}$ & $\begin{array}{c}-3.25 \\
( \pm 0.99)\end{array}$ & $\begin{array}{c}-2.60 \\
( \pm 1.07)\end{array}$ & $\begin{array}{c}2.44 \\
( \pm 0.63)\end{array}$ & $\begin{array}{l}0.9 \\
30\end{array}$ & 0.62 & 17 & $1,2,3,4,5,6,9,1011,12,13,14$ \\
\hline $\mathrm{H}$ & $\begin{array}{c}42.80 \\
( \pm 1.32)\end{array}$ & $\begin{array}{c}-3.95 \\
( \pm 1.46)\end{array}$ & $\begin{array}{c}-2.42 \\
( \pm 1.57)\end{array}$ & $\begin{array}{c}3.09 \\
( \pm 0.93)\end{array}$ & $\begin{array}{l}0.9 \\
07\end{array}$ & 0.91 & 12 & $1,2,3,4,5,6,9,1011,12,13,14$ \\
\hline $\mathrm{Cl}$ & $\begin{array}{c}42.48 \\
( \pm 0.90)\end{array}$ & $\begin{array}{c}-2.56 \\
( \pm 0.99)\end{array}$ & $\begin{array}{c}-3.78 \\
( \pm 1.07)\end{array}$ & $\begin{array}{c}2.22 \\
( \pm 0.63)\end{array}$ & $\begin{array}{c}0.9 \\
11\end{array}$ & 0.62 & 13 & $1,2,3,4,5,6,9,1011,12,13,14$ \\
\hline $\mathrm{Br}$ & $\begin{array}{c}42.52 \\
( \pm 0.88)\end{array}$ & $\begin{array}{c}-1.93 \\
( \pm 0.97)\end{array}$ & $\begin{array}{c}-5.23 \\
( \pm 1.05)\end{array}$ & $\begin{array}{c}3.44 \\
( \pm 0.62)\end{array}$ & $\begin{array}{l}0.9 \\
43\end{array}$ & 0.61 & 21 & $1,2,3,4,5,6,9,1011,12,13,14$ \\
\hline $\mathrm{I}$ & $\begin{array}{c}41.93 \\
( \pm 0.79)\end{array}$ & $\begin{array}{c}-2.37 \\
( \pm 0.87)\end{array}$ & $\begin{array}{c}-3.73 \\
( \pm 0.94)\end{array}$ & $\begin{array}{c}2.04 \\
( \pm 0.56)\end{array}$ & $\begin{array}{l}0.9 \\
20\end{array}$ & 0.55 & 15 & $1,2,3,4,5,6,9,1011,12,13,14$ \\
\hline $\mathrm{COCH}_{3}$ & $\begin{array}{c}38.33 \\
( \pm 0.53)\end{array}$ & $\begin{array}{c}-1.70 \\
( \pm 0.72)\end{array}$ & $\begin{array}{c}-3.95 \\
( \pm 0.54)\end{array}$ & $\begin{array}{c}0.58 \\
( \pm 0.41)\end{array}$ & $\begin{array}{c}0.9 \\
50\end{array}$ & 0.45 & 25 & $1,2,3,4,6,7,8,9,10,12,13,14$ \\
\hline $\mathrm{COOH}$ & $\begin{array}{c}39.76 \\
( \pm 0.82)\end{array}$ & $\begin{array}{c}-1.47 \\
( \pm 0.90)\end{array}$ & $\begin{array}{c}-3.80 \\
( \pm 0.98)\end{array}$ & $\begin{array}{c}2.29 \\
( \pm 0.58)\end{array}$ & $\begin{array}{l}0.9 \\
03\end{array}$ & 0.56 & 12 & $1,2,3,4,5,6,9,1011,12,13,14$ \\
\hline $\mathrm{NO}_{2}$ & $\begin{array}{c}34.13 \\
( \pm 0.15) \\
\end{array}$ & $\begin{array}{c}-2.85 \\
( \pm 0.16) \\
\end{array}$ & $\begin{array}{c}-1.26 \\
( \pm 0.18) \\
\end{array}$ & $\begin{array}{c}0.05 \\
( \pm 0.10) \\
\end{array}$ & $\begin{array}{l}0.9 \\
91 \\
\end{array}$ & 0.10 & 152 & $1,2,3,4,5,6,9,1011,12,13,14$ \\
\hline
\end{tabular}

${ }^{a}$ Correlation coefficient. ${ }^{b}$ Standard error of the estimate. ${ }^{c}$ Fisher's test. ${ }^{d}$ Solvent number as given in Table 2.

Table 4. Percentage contribution of solvatochromic parameters

\begin{tabular}{ccccccccc}
\hline Substituent Z & $\mathrm{P}^{*}(\%)$ & $\mathrm{P}_{\beta}(\%)$ & $\mathrm{P}_{\alpha}(\%)$ & $\begin{array}{c}\text { Substituent } \\
\mathrm{Z}\end{array}$ & $\mathrm{P} \pi^{*}(\%)$ & $\mathrm{P}_{\beta}(\%)$ & $\mathrm{P}_{\alpha}(\%)$ \\
\cline { 1 - 3 } $\mathrm{N}\left(\mathrm{CH}_{3}\right)_{2}$ & 57 & 21 & 22 & & $\mathrm{Br}$ & 19 & 49 & 32 \\
$\mathrm{OH}$ & 22 & 46 & 32 & $\mathrm{I}$ & 29 & 46 & 25 \\
$\mathrm{OCH}_{3}$ & 35 & 38 & 27 & $\mathrm{COCH}_{3}$ & 28 & 63 & 9 \\
$\mathrm{CH}_{3}$ & 39 & 31 & 30 & $\mathrm{COOH}$ & 20 & 50 & 30 \\
$\mathrm{H}$ & 42 & 26 & 32 & $\mathrm{NO}_{2}$ & 69 & 30 & 1 \\
$\mathrm{Cl}$ & 30 & 44 & 26 & & & & \\
\hline
\end{tabular}




\section{Conclusions}

The satisfactory correlation of the ultraviolet absorption frequencies of the investigated $\mathrm{N}$-(4-substituted phenyl)-2,3-diphenylpropanamides 1 with Eq.1 indicates that the selected model gives a correct interpretation of the linear solvation energy relationships of the complex system of the amides in the solvents used. In the case of both solvents and substrates being hydrogen bond donors and acceptors, it is quite difficult to untangle solvent dipolarity/polarizability and hydrogen bond donating and accepting interactions. We have demonstrated that an equation with three solvatochromic parameters $\pi^{*}, \alpha$ and $\beta$ can be used to evaluate the effects of both types of hydrogen bonding and the solvent dipolarity/polarizability effect. The results show that the solvent effect on UV absorption spectra of $N$-(4-substituted phenyl)-2,3-diphenylpropanamides 1 is very complex and strongly depends on the nature of the substituents at the $\mathrm{N}$-phenyl ring.

\section{Acknowledgements}

The authors acknowledge the financial support of the Ministry of Science and Environmental Protection of the Republic of Serbia (project 142063).

\section{Experimental Section}

$N$-(4-Substituted phenyl)-2,3-diphenylpropanamides $\mathbf{1 a , c , d}, \mathbf{g}-\mathbf{j}$ were prepared from 2,3-diphenylpropanoyl chloride and corresponding anilines. ${ }^{13}$ 2,3-Diphenylpropanoyl chloride was synthesized by the reaction of 2,3-diphenylpropanoic acid and thionyl chloride. ${ }^{14} 2,3$-Diphenylpropanoic acid was obtained by the hydrolysis of 2,3-diphenylpropanitrile, which was obtained by the reaction of phenylacetonitrile and benzyl chloride. ${ }^{15}$ The following compounds are known: $\mathrm{N}$-phenyl-2,3-diphenylpropanamide (1e), ${ }^{16} \mathrm{~N}$-(4-nitrophenyl)-2,3-diphenylpropanamide (1k) ${ }^{17} \quad N$-(4-chlorophenyl)-2,3-diphenylpropanamide $\quad(\mathbf{1 f}),{ }^{18}$ and $N$-(4-hydroxyphenyl)-2,3diphenylpropanamide (1b). ${ }^{19}$ 
Table 5. Physical and spectral data of $N$-(aryl)-2,3-diphenylpropanamides 1a-k. ${ }^{a}$

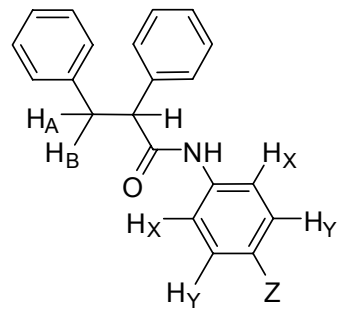

1

\begin{tabular}{|c|c|c|c|c|c|c|}
\hline $\begin{array}{c}\text { Subst. } 1 \\
\text { Z }\end{array}$ & $\begin{array}{l}\mathrm{mp} \\
{\left[{ }^{\circ} \mathrm{C}\right]}\end{array}$ & $\begin{array}{l}\text { Lit. mp } \\
\left.\qquad{ }^{\circ} \mathrm{C}\right]\end{array}$ & $\begin{array}{c}\mathrm{IR}(\mathrm{KBr}) \\
\widetilde{v}_{\max } \\
{\left[\mathrm{cm}^{-1}\right]}\end{array}$ & ${ }^{1} \mathrm{H}$ NMR $\delta[\mathrm{ppm}]\left(\mathrm{CDCl}_{3}\right)$ & $\begin{array}{l}\mathrm{MS} \\
\mathrm{m} / \mathrm{z} \\
\left(\mathrm{M}^{+}\right)\end{array}$ & $\begin{array}{l}\text { Yield } \\
(\%)\end{array}$ \\
\hline $\begin{array}{c}\mathbf{a} \\
\mathrm{N}\left(\mathrm{CH}_{3}\right)_{2}\end{array}$ & $\begin{array}{l}162- \\
164\end{array}$ & & $\begin{array}{l}3266(\mathrm{~N}-\mathrm{H}) \\
1640(\mathrm{C}=\mathrm{O})\end{array}$ & $\begin{array}{l}2.85\left[\mathrm{~s}, 6 \mathrm{H}, \mathrm{N}\left(\mathrm{CH}_{3}\right)_{2}\right], 3.02(\mathrm{dd}, J=6.6,13.1 \mathrm{~Hz}, \\
\left.1 \mathrm{H}, \mathrm{CH}_{\mathrm{A}}\right), 3.74-3.54\left(\mathrm{~m}, 2 \mathrm{H}, \mathrm{CH}, \mathrm{CH}_{\mathrm{B}}\right), 6.61 \\
6.57\left(\mathrm{H}_{\mathrm{YY}}, 2 \mathrm{H}, 3,5-\mathrm{H}_{\mathrm{Ar}}\right), 7.35-7.10(\mathrm{~m}, 12 \mathrm{H} \\
\left.\mathrm{H}_{\mathrm{XX}}, 2 \mathrm{Ph}\right)\end{array}$ & 344.1 & 72.5 \\
\hline $\begin{array}{c}\mathbf{b} \\
\mathrm{OH}\end{array}$ & $\begin{array}{l}160- \\
162\end{array}$ & $\begin{array}{l}101- \\
104^{19}\end{array}$ & $\begin{array}{l}3275(\mathrm{~N}-\mathrm{H}) \\
1647(\mathrm{C}=\mathrm{O})\end{array}$ & $\begin{array}{l}3.13\left(\mathrm{dd}, J=6.2,13.6 \mathrm{~Hz}, 1 \mathrm{H}, \mathrm{CH}_{\mathrm{A}}\right), 3.65-3.41 \\
\left(\mathrm{~m}, 2 \mathrm{H}, \mathrm{CH}, \mathrm{CH}_{\mathrm{B}}\right), 6.45,6.41\left(\mathrm{H}_{\mathrm{YY}}, 2 \mathrm{H}, 3,5-\right. \\
\left.\mathrm{H}_{\mathrm{Ar}}\right), 7.39-7.03\left(\mathrm{~m}, 12 \mathrm{H}, \mathrm{H}_{\mathrm{XX}}, 2 \mathrm{Ph}\right), 8.18(\mathrm{~s}, 1 \mathrm{H}, \\
\mathrm{NH})\end{array}$ & 317.0 & 40.9 \\
\hline $\begin{array}{c}\text { c } \\
\mathrm{OCH}_{3}\end{array}$ & $\begin{array}{l}156- \\
157\end{array}$ & & $\begin{array}{l}3279(\mathrm{~N}-\mathrm{H}), \\
1649(\mathrm{C}=\mathrm{O})\end{array}$ & $\begin{array}{l}3.02\left(\mathrm{dd}, J=6.8,13.3 \mathrm{~Hz}, 1 \mathrm{H}, \mathrm{CH}_{\mathrm{A}}\right), 3.76-3.53 \\
\left(\mathrm{~m}, 2 \mathrm{H}, \mathrm{CH}, \mathrm{CH}_{\mathrm{B}}\right), 3.71\left(\mathrm{~s}, 3 \mathrm{H}, \mathrm{OCH}_{3}\right), 6.74, \\
6.70\left(\mathrm{H}_{\mathrm{YY}}, 2 \mathrm{H}, 3,5-\mathrm{H}_{\mathrm{Ar}}\right), 7.31-7.09(\mathrm{~m}, 12 \mathrm{H}, \\
\left.\mathrm{H}_{\mathrm{XX}}, 2 \mathrm{Ph}\right)\end{array}$ & 330.9 & 33 \\
\hline $\begin{array}{c}\mathbf{d} \\
\mathrm{CH}_{3}\end{array}$ & $\begin{array}{l}158- \\
159\end{array}$ & & $\begin{array}{l}3285(\mathrm{~N}-\mathrm{H}), \\
1649(\mathrm{C}=\mathrm{O}) .\end{array}$ & $\begin{array}{l}2.25\left(\mathrm{~s}, 3 \mathrm{H}, \mathrm{CH}_{3}\right), 3.03(\mathrm{dd}, J=6.8,12.9 \mathrm{~Hz}, 1 \mathrm{H}, \\
\left.\mathrm{CH}_{\mathrm{A}}\right), 3.75-3.55\left(\mathrm{~m}, 2 \mathrm{H}, \mathrm{CH}, \mathrm{CH}_{\mathrm{B}}\right), 7.33-7.00 \\
(\mathrm{~m}, 14 \mathrm{H}, \mathrm{Ar}, 2 \mathrm{Ph}), 8.20(\mathrm{~s}, 1 \mathrm{H}, \mathrm{NH})\end{array}$ & 314.9 & 34.3 \\
\hline $\begin{array}{l}\mathbf{e} \\
\mathrm{H}\end{array}$ & $\begin{array}{l}168- \\
169\end{array}$ & $\begin{array}{l}166^{16 \mathrm{a}} \\
1168- \\
8.5^{16 \mathrm{~b}} \\
169^{16 \mathrm{c}}\end{array}$ & $\begin{array}{l}3085(\mathrm{~N}-\mathrm{H}), \\
1652(\mathrm{C}=\mathrm{O})\end{array}$ & $\begin{array}{l}3.04\left(\mathrm{dd}, J=6.6,13.2 \mathrm{~Hz}, 1 \mathrm{H}, \mathrm{CH}_{\mathrm{A}}\right), 3.77-3.56 \\
\left(\mathrm{~m}, 2 \mathrm{H}, \mathrm{CH}, \mathrm{CH}_{\mathrm{B}}\right), 7.37-7.00(\mathrm{~m}, 15 \mathrm{H}, 3 \mathrm{Ph})\end{array}$ & 300.9 & 59 \\
\hline $\begin{array}{c}\mathbf{f} \\
\mathrm{Cl}\end{array}$ & $\begin{array}{l}168- \\
170\end{array}$ & $\begin{array}{l}168- \\
170^{18}\end{array}$ & $\begin{array}{l}3285(\mathrm{~N}-\mathrm{H}), \\
1652(\mathrm{C}=\mathrm{O}) .\end{array}$ & $\begin{array}{l}3.40\left(\mathrm{dd}, J=6.2,13 \mathrm{~Hz}, 1 \mathrm{H}, \mathrm{CH}_{\mathrm{A}}\right), 3.75-3.55(\mathrm{~m}, \\
\left.2 \mathrm{H}, \mathrm{CH}, \mathrm{CH}_{\mathrm{B}}\right), 7.39-7.00(\mathrm{~m}, 14 \mathrm{H}, \mathrm{Ar}, 2 \mathrm{Ph}), 8.20 \\
(\mathrm{~s}, 1 \mathrm{H}, \mathrm{NH})\end{array}$ & 335.9 & 29.3 \\
\hline $\begin{array}{c}\text { g } \\
\mathrm{Br}\end{array}$ & $\begin{array}{l}171- \\
173 \\
\end{array}$ & & $\begin{array}{l}3283(\mathrm{~N}-\mathrm{H}), \\
1653(\mathrm{C}=\mathrm{O})\end{array}$ & $\begin{array}{l}3.03\left(\mathrm{dd}, J=6.6,13.2 \mathrm{~Hz}, 1 \mathrm{H}, \mathrm{CH}_{\mathrm{A}}\right), 3.76-3.53 \\
\left(\mathrm{~m}, 2 \mathrm{H}, \mathrm{CH}, \mathrm{CH}_{\mathrm{B}}\right), 7.35-7.08(\mathrm{~m}, 14 \mathrm{H}, \mathrm{Ar}, 2 \mathrm{Ph})\end{array}$ & 379.9 & 65.7 \\
\hline $\begin{array}{l}\text { h } \\
\text { I }\end{array}$ & $\begin{array}{l}188- \\
189\end{array}$ & & $\begin{array}{l}3310(\mathrm{~N}-\mathrm{H}) \\
1659(\mathrm{C}=\mathrm{O})\end{array}$ & $\begin{array}{l}3.04\left(\mathrm{dd}, J=6.6,12.8 \mathrm{~Hz}, 1 \mathrm{H}, \mathrm{CH}_{\mathrm{A}}\right), 3.75-3.54 \\
\left(\mathrm{~m}, 2 \mathrm{H}, \mathrm{CH}, \mathrm{CH}_{\mathrm{B}}\right), 7.38-6.99\left(\mathrm{~m}, 12 \mathrm{H}, \mathrm{H}_{\mathrm{XX}}, 2,6-\right. \\
\left.\mathrm{H}_{\mathrm{Ar}}, 2 \mathrm{Ph}\right), 7.56,7.51\left(\mathrm{H}_{\mathrm{YY}}, 2 \mathrm{H}, 3,5-\mathrm{H}_{\mathrm{Ar}}\right)\end{array}$ & 426.9 & 58.5 \\
\hline
\end{tabular}




\begin{tabular}{|c|c|c|c|c|c|c|}
\hline $\begin{array}{c}\mathbf{i} \\
\mathrm{COCH}_{3}\end{array}$ & $\begin{array}{l}124- \\
125\end{array}$ & & $\begin{array}{l}3295(\mathrm{~N}-\mathrm{H}), \\
1663(\mathrm{C}=\mathrm{O})\end{array}$ & $\begin{array}{l}2.49\left(\mathrm{~s}, 3 \mathrm{H}, \mathrm{COCH}_{3}\right), 3.04(\mathrm{dd}, J=7,13.4 \mathrm{~Hz} \\
\left.1 \mathrm{H}, \mathrm{CH}_{\mathrm{A}}\right), 3.86-3.54\left(\mathrm{~m}, 2 \mathrm{H}, \mathrm{CH}, \mathrm{CH}_{\mathrm{B}}\right), 7.40- \\
7.09(\mathrm{~m}, 10 \mathrm{H}, 2 \mathrm{Ph}), 7.49,7.44\left(\mathrm{H}_{\mathrm{XX}}, 2 \mathrm{H}, 2,6-\right. \\
\left.\mathrm{H}_{\mathrm{Ar}}\right), 7.82,7.78\left(\mathrm{H}_{\mathrm{YY}}, 2 \mathrm{H}, 3,5-\mathrm{H}_{\mathrm{Ar}}\right), 8.38(\mathrm{~s}, 1 \mathrm{H}, \\
\mathrm{NH})\end{array}$ & 342.9 & 66.9 \\
\hline $\begin{array}{c}\mathbf{j} \\
\mathrm{COOH}\end{array}$ & $\begin{array}{l}210- \\
211\end{array}$ & & $\begin{array}{l}3283(\mathrm{~N}-\mathrm{H}), \\
1694(\mathrm{C}=\mathrm{O})\end{array}$ & $\begin{array}{l}3.06\left(\mathrm{dd}, J=6.6,13.5 \mathrm{~Hz}, 1 \mathrm{H}, \mathrm{CH}_{\mathrm{A}}\right), 3.75-3.57 \\
\left(\mathrm{~m}, 2 \mathrm{H}, \mathrm{CH}, \mathrm{CH}_{\mathrm{B}}\right), 7.37-7.10(\mathrm{~m}, 10 \mathrm{H}, 2 \mathrm{Ph}) \\
7.451,7.47\left(\mathrm{H}_{\mathrm{XX}}, 2 \mathrm{H}, 2,6-\mathrm{H}_{\mathrm{Ar}}\right), 8.03,7.98\left(\mathrm{H}_{\mathrm{YY}},\right. \\
\left.2 \mathrm{H}, 3,5-\mathrm{H}_{\mathrm{Ar}}\right)\end{array}$ & 345.0 & 66.7 \\
\hline $\begin{array}{c}\mathbf{k} \\
\mathrm{NO}_{2}\end{array}$ & $\begin{array}{l}134- \\
136\end{array}$ & $\begin{array}{l}134- \\
136^{17}\end{array}$ & $\begin{array}{l}3257(\mathrm{~N}-\mathrm{H}), \\
1662(\mathrm{C}=\mathrm{O})\end{array}$ & $\begin{array}{l}3.06\left(\mathrm{dd}, J=6.6,13.8 \mathrm{~Hz}, 1 \mathrm{H}, \mathrm{CH}_{\mathrm{A}}\right), 3.86-3.53 \\
\left(\mathrm{~m}, 2 \mathrm{H}, \mathrm{CH}, \mathrm{CH}_{\mathrm{B}}\right), 7.35-7.09(\mathrm{~m}, 10 \mathrm{H}, 2 \mathrm{Ph}), \\
7.54,7.50\left(\mathrm{H}_{\mathrm{XX}}, 2 \mathrm{H}, 2,6-\mathrm{H}_{\mathrm{Ar}}\right), 8.10,8.05\left(\mathrm{H}_{\mathrm{YY}},\right. \\
\left.2 \mathrm{H}, 3,5-\mathrm{H}_{\mathrm{Ar}}\right), 8.43(\mathrm{~s}, 1 \mathrm{H}, \mathrm{NH})\end{array}$ & 345.9 & 29.4 \\
\hline
\end{tabular}

${ }^{\mathrm{a}}$ The purity of products $\mathbf{1}$ has been proven by GC analysis as $98.5-99.5 \%$.

Amides 1 (Table 5) were recrystallized from ethanol/water. Purity was confirmed by GC analysis on a Varian 3400 gas chromatograph (flame ionization detector, all-glass split-splitless sample injector, 1071 capillary injector, DB-1 capillary column). Data handling by a Varian 4720 data system. Melting points were determined on an electrothermal apparatus. FT-IR spectra were recorded with a Bomem MB 100 spectrophotometer. ${ }^{1} \mathrm{H} \mathrm{NMR}$ spectra of $\mathrm{CDCl}_{3}$ solutions (TMS as internal standard) were measured with a Varian-Gemini $200 \mathrm{MHz}$ spectrometer. MS data were determined with a Polaris MS mass spectrometer (direct probe, $70 \mathrm{eV}$ ionizing energy, emission current of $600 \mu \mathrm{A}$ ). UV absorption spectra were measured with a Shimadzu $160 \mathrm{~A}$ spectrophotometer. The spectra were taken in spectroquality solvents with $10^{-5} \mathrm{M}$ concentration.

\section{References}

1. Valentić, N. V.; Ušćumlić, G. S.; Radojković-Veličković, M.; Mišić-Vuković, M. J. Serb. Chem. Soc. 1999, 64, 149; Chem. Abstr. 1999, 131, 150875.

2. Valentić N.; Ušćumlić, G.; Radojković-Veličković, M. J. Serb. Chem. Soc. 2000, 65, 81.

3. Mijin, D. Ž.; Ušćumlić, G. S.; Valentić, N. V. J. Serb. Chem. Soc. 2001, 66, 507.

4. Valentić, N.; Ušćumlić, G.; Radojković-Veličković, M. Ind. J. Chem. 2003, 42B, 1137.

5. Jovanović, S.; Mijin, D.; Mišić-Vuković, M. Arkivoc 2006, 10, 116.

6. Ušćumlić, G.; Mijin, D.; Valentić, N.; Vajs, V.; Sušić, B. Chem. Phys. Lett. 2004, 397, 148.

7. Mijin, D.; Ušćumlić, G.; Perišić-Janjić, N.; Valentić, N. Chem. Phys. Lett. 2006, 418, 223.

8. Ušćumlić, G. S.; Stojanović, N. D.; Petrović, S. D. J. Serb. Chem. Soc. 1997, 62, 535; Chem. Abstr. 1997, 127, 205245.

9. Ušćumlić, G. S.; Petrović, S. D. Ind. J. Chem. 2002, 41B, 206. 
10. Ušćumlić, G. S.; Krstić, V. V.; Drmanić, S. Ž. Ind. J. Chem. 1998, 37B, 1024.

11. Reichardt, C. Solvents and Solvent Effects in Organic Chemistry, Wiley-VCH: Weinheim, 2004, p 456.

12. Kamlet, M. J.; Abboud, J. L. M.; Abraham, M. H.; Taft, R. W. J. Org. Chem. 1983, 48, 2877.

13. (a) Petrović, S. D.; Stojanović, N. D.; Stojanović, O. K. J. Serb. Chem. Soc. 1986, 51, 395; Chem. Abstr. 1987, 107, 77390. (b) Petrović, S. D.; Stojanović, N. D.; Stojanović, O. K.; Kobilarov, N. L. J. Serb. Chem. Soc. 1988, 53, 633; Chem. Abstr. 1990, 112, 118398.

14. Rising, M. M.; Swartz, K. T. J. Am. Chem. Soc. 1932, 54, 2021.

15. Rising, M. J. Am. Chem. Soc. 1920, 42, 128.

16. (a) Burton, H; Shoppee, C. W. J. Chem. Soc. 1937, 543; Chem. Abstr. 1937, 31, 4298.

(b) Work, D; Bryant, D. R.; Hauser, C. R. J. Org. Chem. 1964, 29, 722. (c) Spasov, A.; Panaiotova, B. Zh. Obshch. Khim. 1965, 1, 1099. Chem. Abstr. 1965, 63, 62840.

17. Janković, V. D.; Mijin, D. Ž.; Petrović, S. D. J. Serb. Chem. Soc. 2002, 67, 373.

18. Mijin, D. Ž.; Janković, V. D.; Petrović, S. D. J. Serb. Chem. Soc. 2004, 69, 85.

19. Kawakami, H.; Hashimoto, K.; Tamoto, K.; Ono, K.; Yamamoto, M. EP 184822, 1986. Chem. Abstr. 1986, 105, 114756. 